\title{
DERIVADOS DE SABUCUS EN LA TOPCNIMIA PENINSULAR
}

Los sucesores románicos de los tipos latinos $\mathrm{s}$ a b u c u s $\mathrm{s} a \mathrm{~m} \mathrm{~b} \mathrm{uc}$ us han llamado recientemente la atención de los investigadores. Dámaso Alonso ${ }^{1}$, Paul Aebischer ${ }^{2}$, Max Steffen ${ }^{3}$ y Gerhard Roblfs ${ }^{4}$ se han ocupado de la cuestión con referencias importantes a la Península Ibérica.

Indudablemente, las formas documentadas remontan a s a bucus, tanto en la toponimia, como en el habla viva, lo que hace pensar en el desconocimiento de $\mathrm{s}$ a $\mathrm{mb}$ b $\mathrm{cus}$ por el latín vulgar hispánico ${ }^{5}$. La búsqueda en los nombres de lugar ${ }^{6}$ no me permite documentar más ejemplos de $-1 n b$ que «Sambucón de las 'Torcas» (Adamuz, prov. de Córdoba) ?.

1 El saico entre Galicia y Asturias. (Nombre y supersticion). RDT $P$, II, I946, págs. 3-32.

2 Les tipes sambucus et sabucus "sureau" et leur répartition dans les langutes romanes. VRo, XII, I951, págs. 82-94.

3 A propósito de un alliculo de Dámaso Alonso. El saúco entre $G a$ licia y Asturias (Nombre y superstición). VRo., XIV, I954, páginas 204-222.

- Die lexicalische Differentiernng der ronanischen Sprachen. Miinchen, I954, págs. 13-14.

5 Vid. AEBiscrer, art. cit., pág. 94.

6 Hago la inquisición en los siguientes repertorios J. M. BAPTISTA, Chorographia moderna do reino de Portugal. Lisboa, i 8;8; J. B. Da Sirva Lopes, Diccionario postal. Lisboa, I89I-I894; MIADoz, Diccionario geográfico. Madrid, I845-I850; Diccionario geogr. postal. Madrid, s. a., y Dicc. corogrifico de España. Miadrid, s. a. [Censo de I940]

7 Se cita en los diccionarios postal y corográfico; $\mathrm{MADOZ}$, Diccionario geográfico, I, pág. $79 b$ (Adamuz) dice: hacia el N. O. [de Adamuz] a 2 I/2 leg. de la v. [corre] el [arroyo] llanado Varas en 


\section{"SABUGO" Y FORMAS AFINES}

Los derivados de $\mathrm{s} a \mathrm{bucus}$ son muy abundantes en Galicia y Portıgal y el área de su influencia penetra en algunas zonas del dominio leonés. Las formas derivadas de este étimo son:

I) Sabugo(s) ${ }^{1}$, Sabugosa(s) ${ }^{2}$, Sabugueira(s) ${ }^{3}$, Sampelayo Sabugueira ${ }^{4}$, Sabugueiro(s) ${ }^{5}$, Sabugueiro de Abajo y de Arriba ${ }^{6}$. Como colectivos, encuentro: Sabugal ${ }^{7}$, Sabuguido $^{8}$, Sabuceda ${ }^{9}$, Sabucedo $^{10}$ y los compuestos San Lo-

cl que se halla una profundidad innensurable conocida con el nombre del Sambucón de S. Torcazs. No deja de ser suyzstiva la coincidencia, si es que Sambucón procede directamente de $s$ a in bu cus, del topónimo con el uso por el cordobés Columela de la voz latina con -MB(vid. Alebisciner, art. cit., pág. 82).

1 Se localiza en $A$ vilés (Oviedo), Castropol (id.), Luarca (id.), Piedrafita (Lugo), Abadin (id.), Arruda (Lisboa), Cintra (idl.).

2 Tondella, dos veces (Viseu), Guimarāes (Braga), Paredes (Porto). Esta forma debe relacionarse con la catalana medieval Saugosa (AEBISCIIER, art. cit., pág. 87).

3 Enfesta (Coruña), Sobrado (id.), Puertomarín (I,ugo), Taboada (id.). Valle de Oro, dos veces (id.), Riotorto (id.).

4 Enfesta (Coruña).

b Capela, Cerceda, dos veces, Lousame, Miño, Noya (todos en La Coruña), Castro de Rey, Castro Verde, Priol, Germade, Sober, Trasparga, Villalba, Vivero (todos en Lugo), Castro Caldelas y Rio (Orcnse), Cambados, La Cañiza, Cotovad (los tres en Pontevedra), Aljustrel (Beja), Celorico de Basto, Guimarães (Braga), Soure (Coimbra), Arraiolos, Benavente, Estremoz, Monte Môr o Novo (todos en Evora), Almeida, Ceia (Guarda), Obidos (Leiria), Lousada, Paços de Ferreira (Porto), Paredes de Coura, dos veces, y Ponte do Lima (Vianna do Castello), Castro Daire, Sinfäes (Viscu).

6 Ambos en Río (Orense).

7 Agueda (Aveiro), Fafe, (Braga), Olleiros (Castello Branco), Sabugal (Guarda), Arcos de Valle de Vez (Vianna do Castello). Falta en Galicia.

8 Villarino de Couso (Orense).

- Boiro y Touro (La Coruña). Zabuceda en Trazo (Morlan, provincia de La Coruña).

$1 \theta$ Muros, Orol, Valle de Oro (Lugo), Baltar, Cartelle, Porquera y Verea (Orense) y Eistrada (l'ontevedra). 


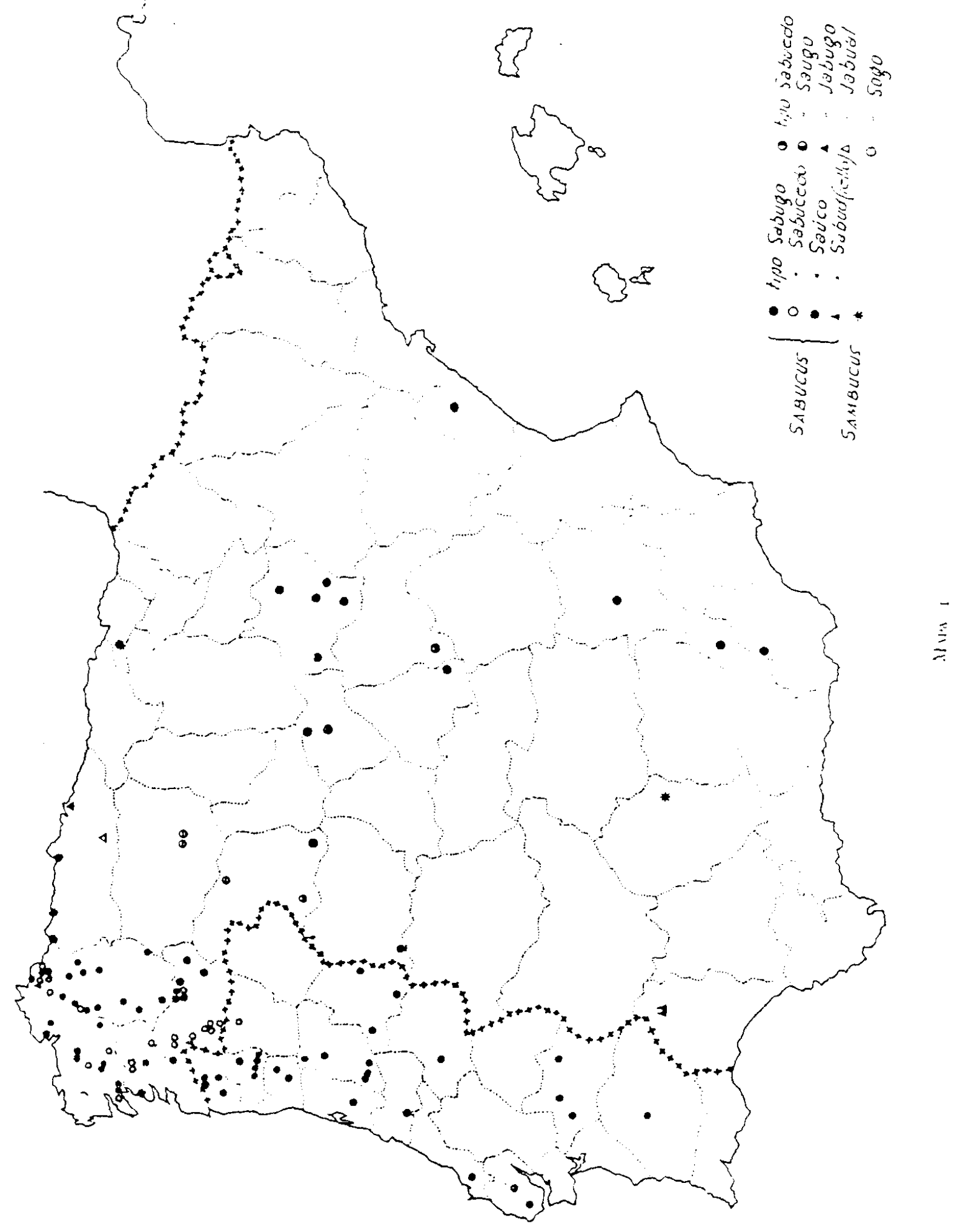



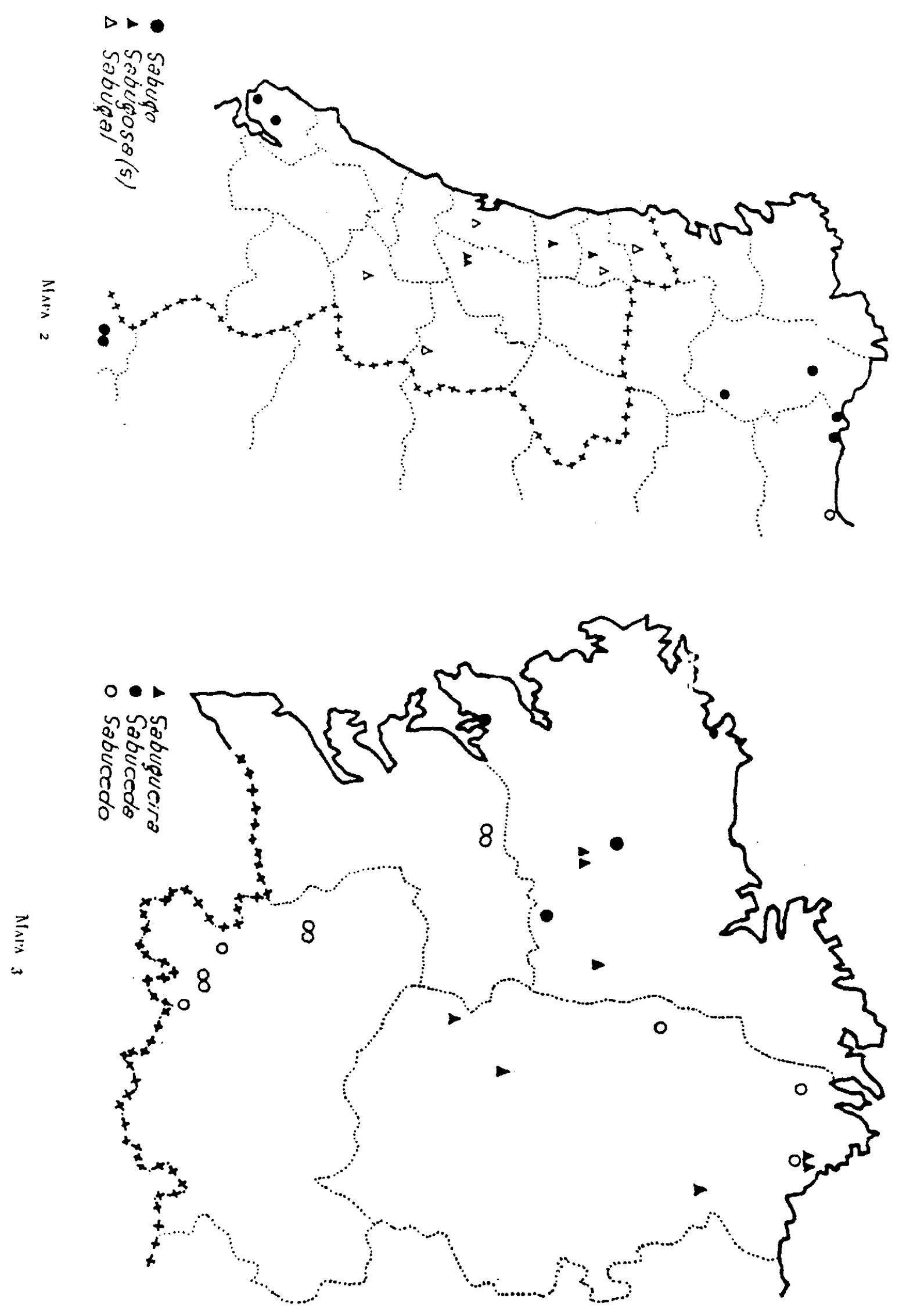
renzo Sabucedo ${ }^{1}$ y San Salvador Sabucedo ${ }^{2}$, San Pedro Sabucedo de Montes ${ }^{3}$.

$\mathrm{S}$ a bu cus da normalmente Sabugo, tanto en la toponimia como en el habla viva. Pero Dámaso Alonso señaló que sabugo se ha ceñido al sentido de 'medula de saúco', mientras que sabugueiro es el 'arbusto que tiene sabugo' 4 ; sin embargo, hoy, algunos pueblos de Lugo (Condes, Corgo, Foilevar, Santa Marina, San Pelayo de Arcos y Santa Eulalia de Sisoy) y uno de Pontevedra (Ventosa) designan con sabugo al arbusto ${ }^{5}$. Así también ocurre en Asturias ${ }^{6}$ de modo general, aunque de las variantes fonéticas del bable me ocuparé más adelante. Señalemos ahora que el arcaísmo sabugo 'saúco' se encuentra en la toponimia y en el habla viva de Lugo y Asturias y que en una época antigua, en la Reconquista del Sur de Extremadura, la voz migró hasta el Norte de Huelva, donde dejó algún resto toponímico ?. En Portugal sólo hay un par de nombres de lugar en el distrito de Lisboa.

Cumplida la dualidad significativa de sabugo (arbusto $>$ medula), el sufijo - a ri u ( $>$-eiro, -ero) vino a resolver la homonimia. Sin embargo, en el dominio gallego (mapa 3) hay que señalar la coexistencia de las formas masculinas $y$ femeninas, aunque la alternancia no consta en los diccionarios (Valladares, Cuveiro, Carré), que recogen sólo sabugıeiro. Así, pues, aunque la toponimia de las dos provincias septen-

\footnotetext{
1 Estrada (Pontevedra).

2 Porquera (Orense).

3 Cartelle (Orense).

4 Art. cit. en la nota I, págs. 3-4. Cfr. "sabugo 'miolo do sabugueiro i sabugueiro'" (Figueiredo, Dicc. Lingua Port., s. v.). En el Dicc. gallego-cast. de VALLADARES, sabugo es sinónimo de sabuguciro, aunque uno de sus valores denuncia ya el cambio semántico: 'la varte huesosa que hay en lo interior de las astas del ganado vacuno y lo iuás vivo de las uñas de toclo animal'.

$5 \quad I b .$, pág. 4 y sus notas 5 y 6 . Nuevos datos er mapa 6.

- En el mapa núıero 2 no señalo más que las localizaciones centrales y occidentales del Principado. Para Asturias, véase el mapa número 4 .

7 De las formas onubenses me ocupo luego, vid. púg. 25.
} 
trionales de Galicia presentan el femenino --aria como sufijo para designar especies vegetales, la forma general masculina ha invadido este reducto ${ }^{1}$.

La voz sabugal aplicada al 'sitio poblado de saúcos' no debe ser muy general en Galicia: el testimonio de la toponimia es harto elocuente (ni un solo caso) y con él están concordes los diccionarios, que no le dan siempre cabida. ${ }^{2}$. En portugués sabemos que la voz significa 'terreno onde crescem sabugueiros' (Figueiredo) y tal debe ser el sentido de los cinco topónimos registrados. Es de notar que el área de la voz es, en toponimia, más restringida que la de sabugueiro (véanse mapas I y 2). Su documentación antigua se aduce en la p. 27.

Dentro del área anterior queda inscrita la de Sabugosa, cuya vitalidad actual debe ser muy escasa pues no figura en los diccionarios gallegos ni, lo que autoriza la suposición, en el abundantísimo de Figueiredo.

El colectivo Sabuguido deriva (por analogía con los frecuentes topónimos en - etum que designan agrupaciones botánicas) tardíamente de Sabugo, ya que de remontar a época latina hubiera dado Sabucedo, como tantos otros. Sin embargo, debe tener cierta antigüedad, mayor sin duda que el cambio semántico sabugo 'arbusto' > 'medula' y, por tanto, anterior también a la aparición del sabugueiro como nombre del arbusto. El cambio fonético -edo $>-i d o$ es frecuente en gallego ${ }^{3}$.

Los colectivos del tipo Sabucedo (port. Sabuzedo) fonéticamente no ofrecen dificultad. La $c(z)$ es el resultado normal de $\mathrm{k}$ latina ante vocal palatal $\left(<^{*} \mathrm{~s}\right.$ a b u c e $\left.t \mathrm{u}\right)$, por eso figura en este grupo aunque la solución de la $\mathrm{K}$ no haya sido la velar sonora.

1 En cada una de estas provincias cuento cinco casos de -eiro(s) por tres (La Coruña) y cinco (Lugo) de -eira.

2 Falta en los de Vali,adares y Cuveiro y figura en el de Carre con estos dos valores: 'mata de saúco. Lugar abundante en saúco'.

3 Vid. V. GARCA DE DIEgo, Dialectologia hispánica. Madrid, 1946, pág. 74, § 22 g. 
Coinciden con este tratamiento de las consonantes intervocálicas, los dos topónimos Jabugo y Jabuguillo del partido de Aracena (Huelva). La misma palatización de la $s$ - inicial, que, en apariencia, separaría los topónimos meridionales de los norteños, viene a unirlos con una región muy concreta de Asturias. Estos hechos hay que condicionarlos a la reconquista leonesa, cuyos frutos duran todavía no sólo en la lengua ${ }^{1}$ sino también en la cultura material ${ }^{2}$ o en el folklore ${ }^{3}$. Si, por la $b$ y la $g$, Jabugo pertenece al oeste peninsular, por su $J$ $(<\breve{s})$ inicial coincide con una precisa zona del asturiano oriental. En el mapa número 4 he pretendido reflejar el tratamiento fonético de la voz en el Principado: me he servido de vocabularios y monografías ${ }^{4}$ por eso la desigualdad en la distribución de los datos ${ }^{5}$. Si es lícito relacionar, comu creo, y he dado

1 Vid. NAVARro TOMás, Espinosa, R. CAsteidano, La frontera del andaluz, $R F E, \mathrm{XX}$, 1933, pág. 234, y R. Ménéndrz PIDAL, El dialecto leonés. $R B A M, \mathrm{XIV}, 1906, \mathrm{p}$. I33. La publicación del Atlas linguiistico de Andalucía permitirá ver una gran influencia lconesa en el léxico del norte de la provincia de Huelva.

2 J. Caro Baroja, Los arados españoles (sus tipos y reparticiones). RDTP, V, I949, figura II 4 (tras la pág. 92).

3 P. GONZALEZ GUZMÁ, Geografía. folklórica: a propósito de la - Balada triste» de Garcia Loxca. Misc. dedic. Griera, I, pág. 3 I I (mapa). Lo que el autor llama zona onubense es, precisamente, la de mayor influencia leonèsa.

4 He aquí su relación: DAMaso Alonso, art. cit., págs. 4-5; G. Alvarez, El habla de Babia y de Laciana. Madrid, 1949, s. v.; V. Garcla REy, Vocabulario del Bierzo, s. v.; M. MENÉNDEZ GARcis, Cruce de Dialectos en el habla de Sisterna (Asturias), RDTP, VI, In5', página 395; J. NEIRA, El habla de Lena. Oviedo, 1955, s. v.; L. RODRíguez-Castellano, La variedad dialectal del Alto Aller. Ovicdo, 1952, página 86, Aspectos del bable occidental, Oviedo, 1954, pág. I27; B. VIGón, Vocabulario dialectológico del Concejo de Colunga. Madricl, 1955. S. v. Todos estos datos junto a los materiales inéditos se inventarian en las págs. 34-45 del presente estudio.

5 En León señalo, sólo, las formas coincidentes con las asturianas. Como es sabido, el paso $s>\check{s}$ tiene una difusión poco clara en todas las hablas peninsulares; sin embargo, el cambio se tuvo como propio del sayagués teatral (de carácter leonés) y hoy es muy fre - 
RFE, XI.I, 1957

algunas razones, el topónimo meridional con los xabugus asturianos, habría que pensar en que esa comarca próxima a Aracena fué repoblada por gentes venidas de la zona de Mieres-Infiesto-Cangas de Onís o de la Extremadura repoblada por asturianos orientales.

Una ojeada sobre el mapa 5 nos permite ver la distribución de los derivados de $\mathrm{s}$ a b u c u s en Andalucía. Prescindiendo de pormenores que no hacen al caso, se puede decir que Almería, Granada, Jaén, sur de Córdoba y Málaga pertenecen al área de sabuco; norte de Córdoba, Sevilla y N. E. de Cádiz a la de saíco; norte de Huelva y alguno de los pueblos onubenses de la frontera portuguesa, a la de $s a(b)$ ugo. Como manifestación específica de castellanismo se debe señalar la presencia de la velar sorda.

El área de sabugo fué mayor, pues, más o menos esporádicamente, aparece en Sevilla y Cádiz. Salico, forma de la lengua oficial, presenta brotes inconexos en el dominio de saico (dos pueblos de Almería, alguno en Málaga).

En Andalucía esporádicamente aparecen otras denominaciones: palo de magué (Sanlúcar de Guadiana, prov. cle Huelva), cachorro (Mijas, prov. Mrálaga). En dos pueblos de Córdoba (Villaharta y Santa Eufemia) se le llama mariselva, que es otra planta, y, en el norte de Huelva se encuentra, inesperadamente, la designación taquera con que se nombra al saúco en Atajate (Málaga). Existen deformaciones debidas a etimología popular o a equivalencias acústicas (chabuco, trabuco, azalígo, zacuco), circunscritos siempre a un solo pueblo ${ }^{1}$.

cuente en ese dominio (vid. A. Alonso, Trueques de sibilantes, NRFH, I, I947, pág. 4). La palatización de la $s$ - $>\check{s}$ - es un ejemplo más que aducir a los muchos del dialecto; en cuanto a la clistribución geográfica de sabugo / xabugo hay que limitar las palatizaciones del latín s a bucus en el centro-oriente de Asturias, aunque el fenómeno como tendencia fonética goce de cierto arraigo en todo el dominio leonés.

1 Todos estos materiales proceden del Atlas lingütstico de Andalucia, que preparo. Me he limitado a seũalar las discrepancias léxicas -o fonéticas con carácter diferenciador-. El ceceo o la naturaleza de 
P. Aebischer ha recogido las formas que adopta s a b u cus en los documentos medievales ${ }^{1}$. En la región de Oviedo se atestiguan Sabugo (en I028, II32 y Ir33) y Sabuco (en I062), forma ésta que debe juzgarse como cultismo. E1 mismo Cartulario de San Vicente de Oviedo vuelve a repetir otro topónimo llamado Sabugo en I229 (pág. 306), lo mismo que había transcrito Sabuco en 1058 y ro82 (piiginas 67 y 96). A. C. Floriano ha documentado en Asturias otros varios casos coincidentes con la dialectología actual: "In valle de Sabugo est hereditas de Corias" (I20r), "alian terram ad illo sabugo" (III7), "per illa regaria de illo Sabugo" (IrOI), "intro illos terminos de illo Sabugo» (IIOI) ${ }^{2}$, y en un texto del 8I6, Saucum, forma que no creo que nunca haya sido asturiana ${ }^{3}$. Al dominio linguiístico leonés perteneció el antigno monasterio de Vega en cuya jurisdicción se encontraba el "Val de Sabugo" (Irig) ${ }^{4}$.

Las formas portuguesas quedan también atestiguadas por el profesor Aebischer ${ }^{5}$ : Salugosa $(924,921$ ), Samugales (1086) y azanbugeiros (ro86) ${ }^{6}$. Quiero llamar la atención hacia el hecho de que Sabugosa, forma que acaso no exista en el habla

la $s$ no tienen ningún reflejo en la distribución de formas (he llevalo a cabo la comparación sin resultado positivo; por eso no complico el mapa con trazos que nada dicen a mi interés actual). I a cruz que figura en muchos sitios indica que en ellos cl arbusto es desconocido o que no obtuve respuesta.

1 Art. cit., pág. 87.

2 Los cjemplos figuran en las págs. 64,78 y 160 del Libro Registro de Coricts, I. Ovicdo, 1950.

3 Diplomática española del pertodo astur. Oviedo, I949, t. I, página 142.

4 L. Serrano, Cartulario del Monasterio de Vega. Madricl, ig27. página $4 \mathrm{I}$.

5 Art. cit., pág. 88.

- Un botánico árabe del siglo XI o XIJ dice que el satico uabunda en la región de Santarén, pero sus datos no son confirmados por la toponimia (vid. M. Asin, Glosario de voces romances. Madrid-Granada, 1942, pág. 260, núm. 496). 
viva, está acreditada en documentos cuya antigüedad supera los mil años; es bien de notar que la toponimia haya conservado sin olvido durante un milenio la voz condenada a muerte por las hablas vivas. Asimismo es de señalar la gran antigüedad de la forma incrementada, azambugueiros, presunto cultismo ${ }^{1}$, antecedente directo del actual sabugueiro de Portugal, Galicia, Asturias y León ${ }^{2}$.

Por último, en relación con este grupo, debo aducir el Sango de Ciudad Rodrigo ( $\mathrm{s}$ a b u $\mathrm{cu}>$ sabugo $>$ sango), perfectamente adecuado a los dialectalismos salmantinos de hoy saoguero, sauguero y sayugo ${ }^{3}$ (con $-y$ - antihiática), y lcs sogo de Zamora (Sogo, Bi ime de Sog) y Valdesogo de la prcvincia de León ${ }^{4}$.

2) Un grupo de formas procedentes de $\mathrm{s} a \mathrm{bucus}$, limitado también al $\mathrm{N}$. O. ibérico, presenta $d$ en vez de $g$. El cambio, no documentado en la Edad Media, se produce por equivalencia acústica y da lugar a las siguientes formas: $S a$ budiello ${ }^{5}$, Sabuedo ${ }^{6}$ y, acaso, Sabua o Sabuz ${ }^{7}$.

1 Ib., pán. 93.

2 No debe confundirse Azambugciros ( $<\mathrm{s}$ a bucus) con los topónimos actuales, Azambujcira, Zainbujeira y otras formas relacio-

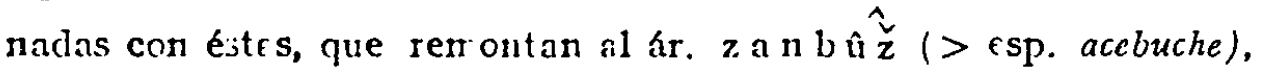
confróntese SiejGrR, Contilución a la fenitica del hispano-ärabc. Madrid, 1932, pág. 194.

3 J. Lamano, El dialecto vulgar salmantino. Salamanca, 1915, página 627 (localizados los dos primeros en Ciudad Rodrigo y el último en la Sierra de Francia). Todos estos términos $\sin -b$ - y con $-k$ - sonorizada tienen algún precedente medieval en la "Garganta de Sahugal" del Libro de la Montería de Alfonso XI (cdic. GuTIÉRREZ DE LA VEGA, Madrid, I877, t. II, p. 42).

4 Este topónimo se llamaba en la E.dad Media ualle de Sabugo (año 937, f. 415r: 960, f. 411 v.; 96r, f. 4 I Ir; 963, f. 415 r.) y, por excepción, ualle de Sabuco ( 965 , f. 4 r 4 r.). Todas estas referencias proceden del Tumbo de la Catedral de León. Mi amigo D. EMm.o SÁzz ha transcrito el códice y me ha pernitido consultar su trabajo.

5 Villaviciosa (Oviє do).

6 Orol (Lugo).

7 Cartelle (Orense). Sabud en el Diccionario postal; Sabuz en el estadistico. 
Sabuedo debe remontar a *Sabudedo, ya que la pérdida de la $g$ entre $e$-o no consta en gallego ${ }^{1}$, mientras que es general la de la dental sonora intervocálica ${ }^{2}$. Desde $*$ Sabudedo, por falsa descomposición, se pudo originar Sabud.

\section{"SAÚCO\# Y FORMAS AFINES}

La voz sanico del español presenta la anomalía de su $c$ mantenida. Meyer-Lübke ${ }^{3}$ la explicó pensando en que fuera una castellanización del catalán sauc, hipótesis abandonada por el gran comparatista que - de modo inexplicable- ha borrado el español saúco en la tercera edición de su REW García de Diego ${ }^{4}$ pensó en que esta $c$ se debia a "una falsa relación con el suf. -uco -u c c u» y su opinión fué aceptada por Dámaso Alonso ${ }^{5}$. Menéndez Pidal ${ }^{6}$ consideró la voz como culta, pero Aebischer ${ }^{7}$ ba desechado la hipótesis con razones bastante sólidas, $\mathrm{y}$ ha propuesto, buscando apoyo en otros investigadores, una nueva explicación: "Sarico aurait son origine dans cette partie du nord de l'Espagne prinitivement occupée par les Basques [se refiere al pirineo centrooccidental]: et ce serait de là qu'il aurait, participant lui aussi à la Reconquista, pénêtré de plus en plus vers le sud, jusqu'à s'introduire dans la langue littéraire» ${ }^{8}$. Esta sugestiva hipótesis ofrece, sin embargo, algunas dificultades:

I) En primer lugar, la región pirenaica donde la $c$ se ha conservado, no es zona en la que el saúco prospere, según puede verse en el mapa número 5 . La escascz del arbusto se re-

1 V. Garcia DE DmGo, Dialectologia hispánica, págs. 56-57.

$2 I b$., pág. 56.

3 REV, $756 \mathrm{x}$.

4 Contribución al diccionario hispánico etimológico. Mradrid, 19+3. número 530 (reimprime casi sin alteraciones la edición de 1923).

5 Art. cit., pág. 5.

6 Manual de gramálica histórica española, $\S 4^{n}$, pág. I29, nota.

7 Art. cit., pág 90.

$8 \quad$ Ib., págs. 92.-93. 
fleja en su limitada documentación en las monografías sobre las hablas pirenaicas; falta, por ejenıplo, en los trabajos de El$\operatorname{cock}^{1}$, González ${ }^{2}$, Alvar ${ }^{3}, y$ en los diccionarios de Badía 4 Borao, Coll ${ }^{5}$ y Pardo $^{6}$, aunque se recoge en las obras de Kulın, Rohlfs ${ }^{7}$ y Badía ${ }^{8}$. La planta es propia de la región galaico-asturiana, y de ella, mejor que del Pirineo donde no abunda demasiado, podría haber adquirido Castilla la denominación del saúco. Vemos, sin embargo, que esto no ha sido así y el castellano nada debe al dominio leonés; por tanto, si las regiones donde más abunda el arbusto, no dieron a Castilla la denominación del saúco no parece lógico que se la facilitaran aquéllas donde escasea. Además, los hechos históricos

1 De quelques affinilés phonétiques entre l'aragonais et le bearnaisIaris, 1938. Fis in:portante esta ausencia porque Elcock estudia la con servación de las oclusivas sordas a ambos lados del Pirineo. Tampoco figura en la alundantisima Toponimia menor en el Alto Ava! ón del mi:mo investigador ("Actas I Rcunión Top. Pirenaica". Zaragoza, 1949, págs. $77^{-118} 8$.

2 El habla viva del valle de Aragücs. Zaragoza, I953.

3 El habla dcl Campo de Jaca. Salamanca, 1948, y Toponimia del allo valle del rto Aragón. Zaragoza, i 949.

4 Contribución al tocabulario aragonés moderno. Zaragoza, I948.

5 Ambos en la scgunda edicićn del Diccionario, de Borso. Zaragoza, 1908. La voz aparcec, como sauquero, en el Vocabulario de

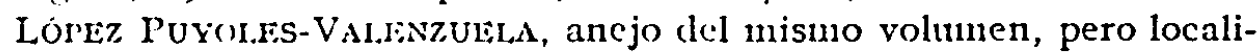
zada ćn el Bajo Aragón.

6 Nucvo diccionario etimológico aragonćs. Zaragoza, 1938.

7 A. Kunn, Der hocharagonesische Dialekt. Leipzig, 1936, páginas 215 y 225, y G. RoHLFs, Le gascon. Halle, 1935, pág. 85, s. v. $\mathrm{s}$ a (111) b u c u in. Las formas y localizacioncs acreditadas por estos investigadores son: sabuco (Ansó, Hecho), sabuquero (Ansó, Hecho, Fanlo, acaso sca la forma más extendida), sauqué (Panticosa), sabucar 'sitio donde hay saucos' (Heclio).

8 El habla del valle de Bielsa. Barcelona, 1950, s. v. samuco 'flor de saúco', samuquer 'saúco'. Para la vertiente francesa, véanse: Ronlls, loc. cit.; J. RonjaT, Grammaire Istorique des parlers provencaux moderns, Montpellier, 1950-1941, §§ 195, 198, 288 y J. SúcuY, Les ncms populatres des flantes dans les Pyrénées centrales. Barcelona, 1953, §§ 158, 159, págs. 373-375, y Allas. 
hubieran ayudado al préstamo leonés y no al aragonés, dadas las relaciones medievales de estos reinos con el central.

2) Del dominio aragonés sólo conocemos las formas modernas ${ }^{1}$ y éstas por su - $b$ - o por su terminación, -ero, se oponen a la hipótesis del aragonesismo .No se puede admitir el préstamo de la $-c-$ y no el de la $-b$ - o el del sufijo ${ }^{2}$.

No me parece mucho más viable que ésta la etimología aceptada por varios autores sarico $<\mathrm{s}$ a b u cus $+-\mathrm{u} \mathrm{c} c \mathrm{u}$. La difusión del sufijo no debió ser muy general en una época, antigua ya que su limitadísima geografía actual no autoriza a otra suposición; me parece difícil que un sufijo poco frecuente influyera sobre una voz bastante conocida. Por otra parte, no alcanzo las causas que obligaron a la confusión (he pensado en que la acción del sufijo pudiera haberse producido en zonas donde salice se convierte en sau, pero evolucionando normalmente $\mathrm{s} a$ (b) $\mathrm{u} \mathrm{cu}>\mathrm{sa}$ (b) ugo se hubieran también evitado los riesgos de una falsa descomposición. De otro modo: que en estas zonas se hubiera creído que era fácil la confusión de sau 'sauce' y saugo = sau + - $\imath$ go).

Lo poco convincente de todas estas hipótesis me hace pensar en que la voz pueda ser, en castellano, un mozarabismo. Según un botánico anónimo del siglo $\mathrm{xr}$ o del xir, šabüq, sabuco eran formas conocidas en la aýamyya ${ }^{3}$. El mozarabismo podría estar favorecido por el prestigio de los médicos y boticarios de Al-Andalus y el uso que hicieran del arbusto en sus recetas.

1 No hay ni un solo topónimo en las tres provincias, ni aparece en las monografías citadas en las notas I-3 de la pág. 30, donde se agrupan abundantes materiales de toponimia menor. Es curioso que la voz falte también en los inventarios de Serrano y Sanz, a pesar de haber publicado el de algún boticario y usarse la flor del saúco en la famiacopea (vid. el Elude Lexicologique de estos inventarios llevado a cabo por B. Potrier, VRo, X, págs. 87-219).

2 En Navarra, al arbusto se le llama sabuco (J. M. IRIBARREN, Vocabulario navarro. Pamplona, 1952, s. v.).

3 M. AsfN, Glosario de voces romances. Mradrid-Granada, I942, número $\mathrm{r} 30$. 
Los derivados de $\mathrm{s}$ a (b) $\mathrm{u}_{\mathrm{c}} \mathrm{u} \mathrm{s}^{1}$ se extienden sin gran densidad por toda España (faltan, naturalmente, en la zona galaico-asturiana ya considerada) y tienen su frecuencia mayor en la provincia de Soria. Copio las formas y localización de estos topónimos: (El) Sanico ${ }^{2}$, Los Saúcos ${ }^{3}$, Sauquillo de Alcázar, Sauquillo de Boinices, Sauquillo del Campo (Adradas), Sauquillo de Paredes (Burgo de Osma) ${ }^{4}$, Sauquillo de Cabezas ${ }^{5}$, Fuentesauico ${ }^{6}$, Fuentesaúco de Fuentidueña ${ }^{7}$.

Hay que tener en cuenta las posibles igualaciones producidas por la lengua oficial: en Cúllar-Baza, el lugarejo se llama Sabuco entre la gente de la localidad; El Saúco de Castellón difícilmente podrá tener esa forma, ya que en catalán el arbusto se denomina sauc, sauquer, sauquera o bonabre ${ }^{8}$, ninguna de las cuales parece haber dejado reliquias en la toponimia catalana.

E1 sauquillo castellano suele denominar al 'yezgo' (Sambucus ebulus), lo mismo que ocurre enl gallego (sabuguiĩo, Carré) y portugués (sabugueirinho, Figueiredo), donde las formas diminutivas son equivalentes de engo(s). El contenido semántico de las voces queda aparte de mi interés actual.

1 S a u c u s se atestigua en GREgorio DE TOURS, Hist. Franc., IV, 9, págs. 147-21, y, según el CGL, III, 590, 5 I, en el siglo $x$.

2 Alcóntar (Almeria), Alhóndiga (Guadalajara), Cúllar (Granada), Galgamés (Vizcaya), Peñas de San Pedro (Albacete) y Sierra de Engarcerán (Castellón de la Plana).

3 Valleseco (Las Palmas de Gran Canaria). Sin embargo, en el habla viva sólo recogi saugo, en Tenerife. Viera da sabugo como forma vulgar (Dicc. hist. natural de las islas Canarias, II, pág. 272).

4 Los cuatro en Soria.

5 Segovia.

- Renieblas (Soria), Valdaracete (Madrid) y Zamora.

7 Cuellas (Segovia).

8 F. MASCLANS, Els noms vulgars de les plantes a les terres catalanes. Barcelona, 1954, pág. 24r b. A lo largo de este trabajo no cito otras variedades que las de Sambucus nigra y Sambucus racemosa; la conocida por Sambucus ebulus presenta - salvo el caso que aclaro inmediatamente-derivados que nada tienen que ver con $s$ a b u $c$ u $s$. 


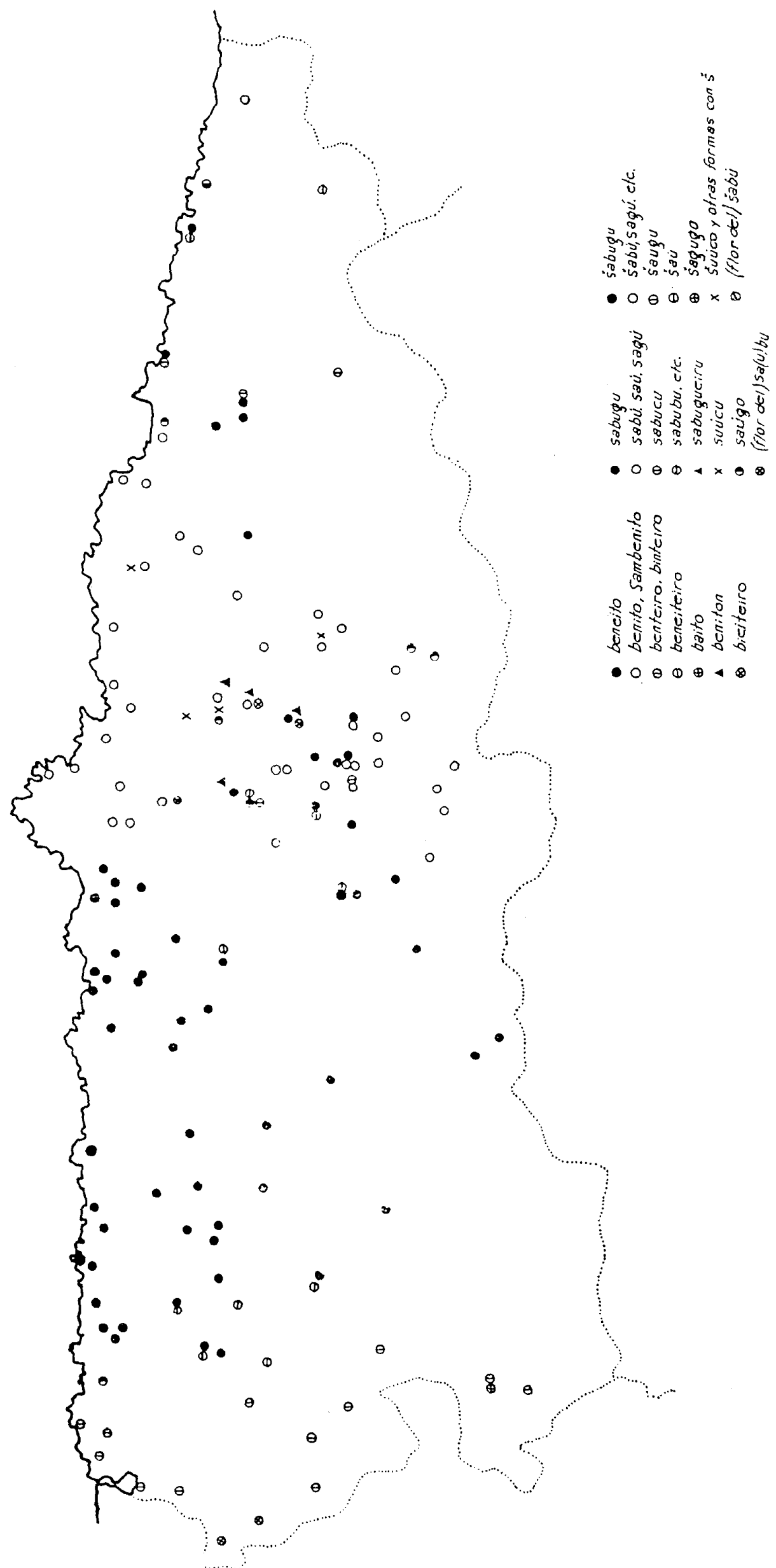




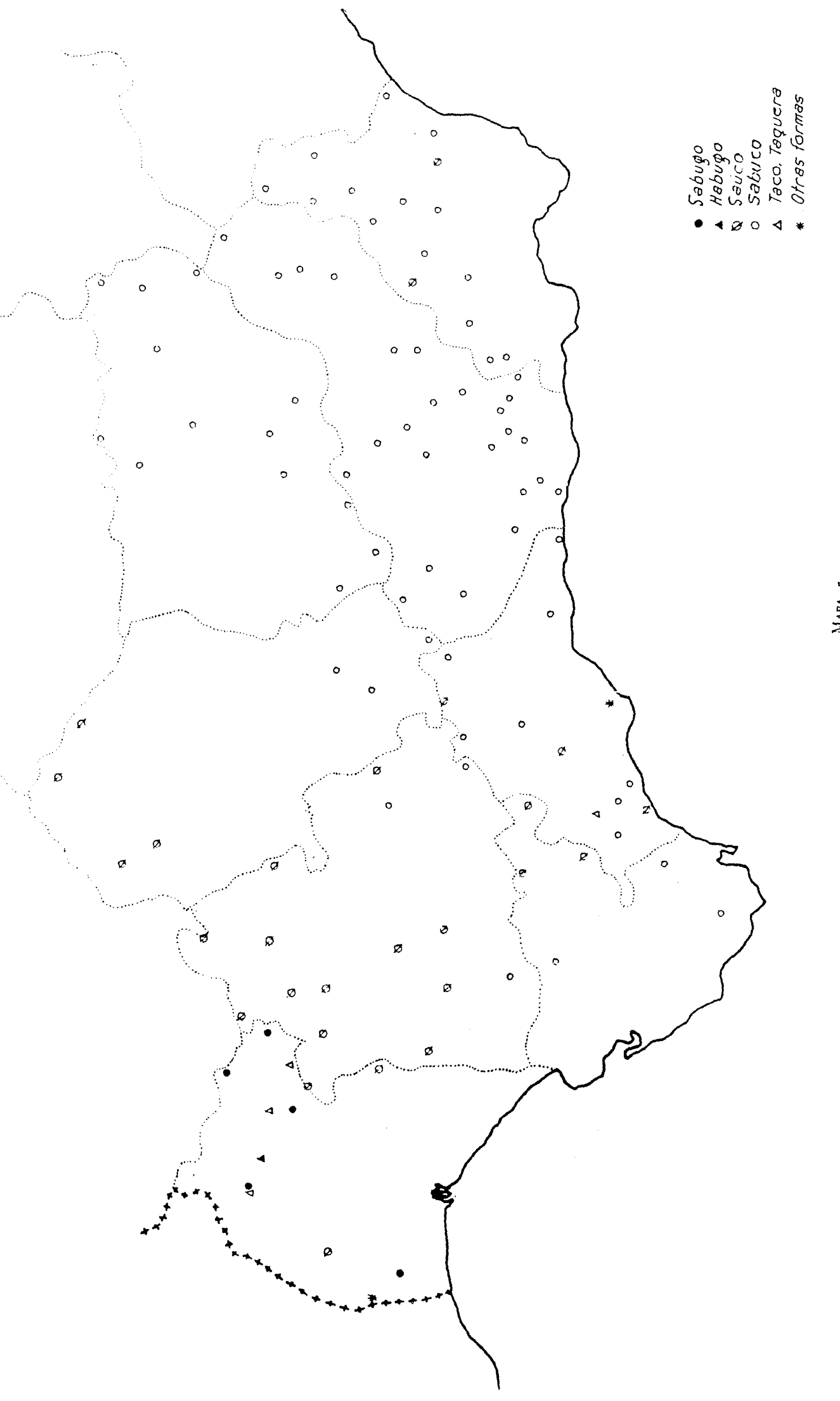


Todas estas formas peninsulares no ofrecen particularidades dignas de mención. El profesor Aebischer señaló ${ }^{1}$ las formas medievales del tipo Sauco (San Pedro de Arlanza), Sabucco, Sabucho (San Millán de la Cogolla) 2; en otras fuentes he encontrado alguna otra con que acrecentar la lista: "Los Saunquiellos" (I085) " "Ualle Sabuco" (IIIg) ${ }^{4}$; Saucum, en una copia de un documento asturiano que se pretende del 860 , pero que, dada la falta de $b$, no creo que pueda ser del Principado ${ }^{5 ;} ; \xi a b \bar{u} q$, ; $\xi^{\prime} a b u c o$ en un botánico árabe ${ }^{6}$.

\section{CONCLUSIONES}

Los topónimos derivados de $\mathrm{s}$ a b u cus dividen a la Península Ibérica en dos zonas perfectamente definidas: una, occidental (Galicia-Portugal-Asturias), y otra. centro-oriental. La primera presenta algunas irradiaciones, como era de esperar, en el dominio leonés o en zonas cuya reconquista es de suponer que fuera leonesa. La zona centro-oriental es mucho más pobre en denominaciones de este tipo ${ }^{7}$. La toponi-

1 Art. cit., págs. 91-92.

2 He aqui las referencias que yo he recogido en los documentos de San Millán: Sabuco (936, pág. 36), Sabucco (ro33, pág. 120), Sabucho (1022, pág. 102 y 1062, pág. I79).

3 J. DEL ALAMO, Colección diplomática de San Salvador de Oña, t. I. Madrid, I950 p. I20.

- V. V[IGNAU], Cartulario del Monasterio de Eslonza (9I2r399). Madrid, I885, pág. 94.

5 A. C. Floriano, Diplomálica astur, ya citada, pág. I 42.

- M. Asfi, Glosario, aducido anteriormente, números i 30 y 496.

7 La fitogeografía ayuda poco a fijar las áreas lingülsticas de cada tipo. El Sambucus Ebulus L. se da ren terrenos cultivados e incultos de las provincias septentrionales, centrales, orientales y meridionale's mientras que el $S$. nigra $L$. prospera en terrenos montuosos y húmedos de las provincias septentrionales, centrales, orientales y meridionales. (ambas referencias proceden de las.págs. I y 3, respectivamente, de la obra de M. Colmerro, Enumeración y revisión de las plantas de la Peninsula hispano-lusitana e islas Baleares. t. III, Madrid, 1887). 
mia coincide perfectamente con las hablas vivas y permite algunas precisiones de carácter histórico.

Un esquemático resumen de las páginas anteriores debera fijarse en los siguientes extremos:

I) En Lugo, Oviedo y norte de Huelva, la toponimia se muestra de acuerdo con la complejidad dialectal de estas regiones.

2) Sabugueira, en vez de sabugueiro, queda circunscrito a las provincias de Lugo y La Coruña. Es, posiblemente, un arcaísmo reducido a la zona norte del dominio lingüístico occidental.

3) Sabugosa es otro arcaísmo salvado por la toponimia y constreñido en el norte de Portugal. Sabemos que existía hace más de mil años, pero hoy ha desaparecido de las hablas vivas.

4) Sabugal sólo se recoge en la toponimia portuguesa; al parecer, es término más frecuente en el país vecino que en Galicia.

5) El Jabugo de Aracena (Huelva) remonta a la época de la Reconquista $y$, en última instancia, procede probablemente del centro-oriente de Asturias, única región donde la $s$ - de s a b u c us pasa a $\xi$-. Naturalmente, a Huelva pudo llegar la forma a través de la Extremadura leonesa.

6) Sarico en castellano no puede ser un aragonesismo.

7) Sabucus es la única forma acreditada por la toponimia; tan sólo hay un Sambucón en Córdoba que, dado su aislamiento, acaso no remonte a $\mathrm{s}$ a $\mathrm{mb} \mathrm{u} \mathrm{cus}$.

\section{A P E N D I C E :}

\section{LAS DESIGNACIONES DEL 'SAUCO' EN LAS HABLAS DEL NOROESTE ESPANTOL}

Gran parte de los materiales que ordeno a continuación han sido utilizados en las páginas de este trabajo. Otros, sin embargo, no han sido aducidos porque su mención era inne- 
cesaria dentro de los límites propuestos. Me parece conveniente dar, a guisa de apéndice, los materiales que tengo allegados sobre el tema porque podrán servir a algún estudioso.

Para la ordenación de los datos que acompaño, he tenido en cuenta la bibliografía que cito en diversas notas; me ha sido de especial utilidad el bello estudio de Dámaso Alonso, cuya aportación dialectal sirvió de base a la lista y al mapa de Max Steffen. Mis materiales, como puede verse, son de una extraordinaria riqueza y complejidad. Debo hacer constar que proceden integramente de datos que con paciencia y perseverancia recogieron para este estudio mis amigos Josefina Casaprima, Angel R. Fernández (materiales gallegos especialmente) y Manuel Menéndez (Asturias): para los tres mi gratitud más honda.

Unas advertencias: en todos los pueblos que transcribo, la palabra designa al arbusto y no a su medula, al sitio poblado por ellos ni a la flor. Si hay alguna discrepancia con este hecho, la consigno en nota.

Entre paréntesis figura el ayuntamiento al que pertenece la localidad.

E1 timbre de la vocal final se transcribe según las indicaciones de los informadores.

\section{abeneito.}

Asturias: Forcinas (Pravia), Puentevieja, Somado (Muros de Nalón).

abieiteiro.

L u g o: Puente Nuevo, Villaodrid.

areiteiro.

Or ense: Aldea de la Bola.

\section{baiteiro.}

Asturias: Pradecís (Ibías), Villamayor (Ibías). 
baito.

Asturias: Pradecís (Ibías), San Antolín de Ibías.

bandieiro.

L u g o: Castelo (Cervantes).

bauteiro.

I u g o: Región de Cervantes.

\section{beneiteiro.}

Asturias: Bustantigo (Allande), Erías (Pola de Lena), Folgueras (Coaña), Len de Quintana (Villayón), Monón (Allande), Ponticiella (Villayón), Rebollo (Allande), Villayón.

beneito.

Asturias: Andés (Navia), Avilés, Castrillón, Collada ('Tineo), Cudillero, Erías (Villayón), Folgueras (Coaña), Forcinas (Pravia), Illas, Jarrio (Coaña), Len de Quintana (Villayón), Miño (Tineo), Miranda, Mohías, (Coaña) Naraval ('Tineo), Naveces (Castrillón), Navelgas (Tineo), Otur (Luarca), Paredes (Luarca), Piedras Blancas (Castrillón), Pillarmo (Castrillón), Ponticiella (Villayón), Puentevega (Pravia), Querúas (Luarca), Rellanos ('Tineo), Riocastiello ('Tineo), San Cristóbal (Luarca), San Feliz (Luarca), San Martín de Luiña (Cudillero), San Miguel (Piloña), Villapedre (Navia), Villayón.

beneuto.

Asturias: Cudillero.

benito.

Asturias: Albandi (Carreño), Amandi (Villaviciosa), Ambás (Villaviciosa), Bimenes, Breceña, (Villaviciosa), Cabra- 


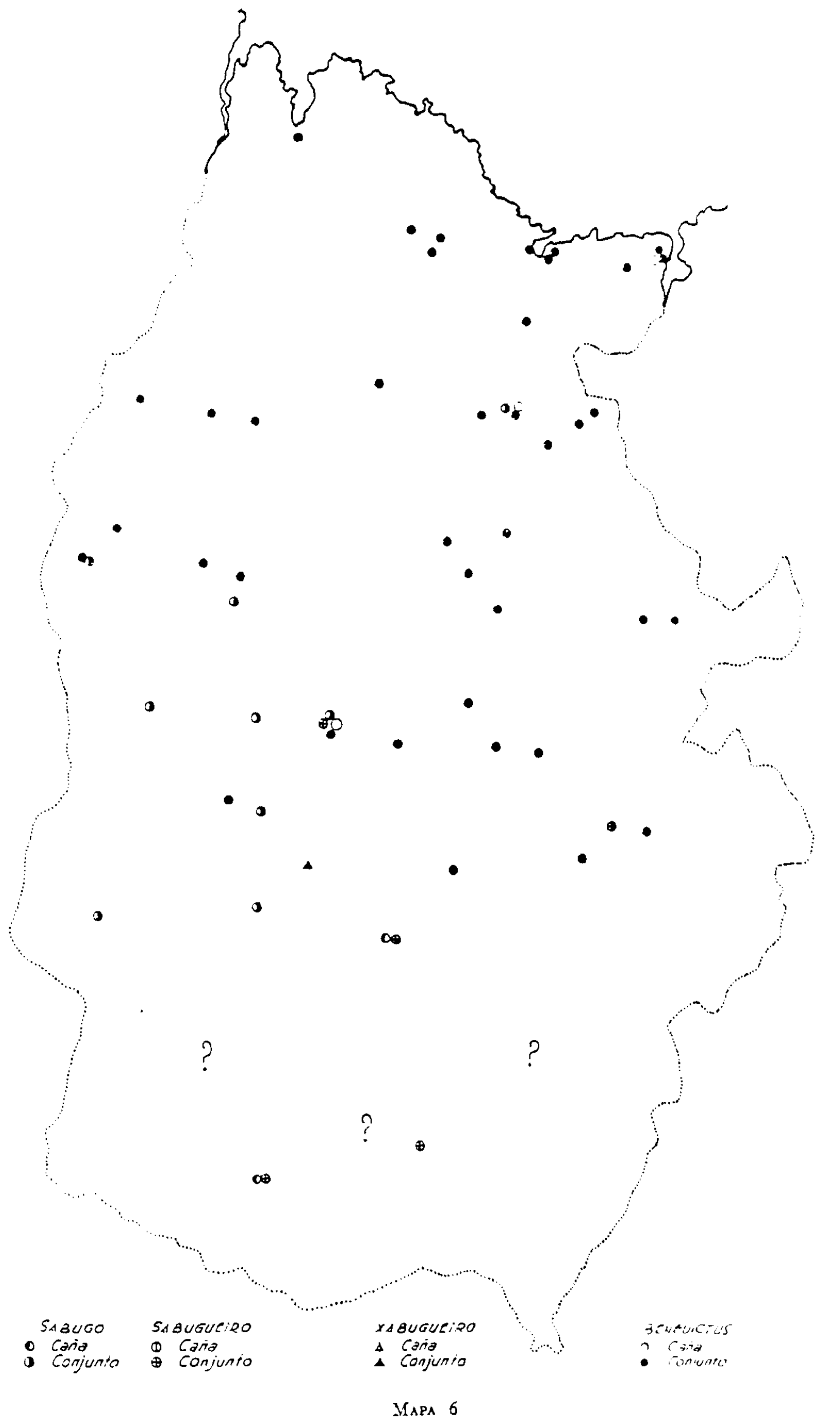


biauteiro.

Cor u ̃̃ a: Coruña, Laracha, Suevos (Arteijo), Villanueva de Serantes.

L u g o : región de Cervantes, Foz, Germade.

Pontevedra: Sotomayor.

L e ó n: Balboa.

\section{bieiteiro.}

C or u ̃̃ a: Bemantes (Miño), Betanzos, Celas de Perio (Culleredo), Cedeira, Valdoviño, Verdillo (Carballo).

L u g o: Abadín, Andión (Pol), Caraño (Pol), Castro del Rey, Cogela (Ribadeo), Fonsagrada, Goiriz (Villalba), Gomesende (Pol), Guitiriz (Trasparga), Meira, Mourence (Villalba), Pigara (Villalba), Remosende (Villalba), Riobarba, Riojuan (Pol), Riotorto, San Pelayo de Arcos (Castroverde), Trabada, Valle Oro, Villaodrid.

P o u t e ved ra: Mondariz, San Vicente de Nogueira (Meis).

Asturias: San Antolín (Ibías), Taramundi.

bieito.

Cor u ñ a: Corcubión.

bieteiro.

Cor u ̃̃ a: Ledoño (Culleredo).

L u g o: Benquerencia (Barreiros).

bleuteiro.

Cor u ñ a: Betanzos, Coruña.

Pontevedra: Pontevedra. 
RFE, XLI, 1950 DERTVADOS DE "SABUCUS" EN LA TOPONIMIA...

bineito.

Asturias: Sisterna (Iblas).

binteiro.

Cor uñ a : Montrove (Oleiros), San Martín de Ponto (Cabanas).

I, u g o: Ribadeo, Luoso (Lugo).

A s t u r i a s : Barres (Castropol), Boal, Brañamayor (El Franco), Castropol, Merou (Boal), Pradecis (Ibías), Prelo (Boal), Rozadas (Boal), San Martín de Oscos, Santa Marfa del Monte (Tapia), Sarzol (Allande), Tol (Castropol), Vegadeo.

bioteiro.

I u g o : Pol, Ríotorto.

biouceiro.

I, u g o: Lugo.

biouteiro.

Cor u ̃̃ a: Arteijo, Enfesta, Jallas, Laracha, Sada.

L u g o : Baralla (Neira de Jusá), Becerreá, Fonsagrada, Germade, Miranda (Castroverde), La Muiña (Baleira), Navia de Suarna, Parga (Trasparga), Queizán (Corgo), San Cosme (Barreiros), San Miguel de Piedrafita (Corgo), Santa Marta de Cerdeiras (Begonte), Santalla (Lugo), Santiago de Cedrón (Láncara).

bouteiro.

Cor u ̃̃ a: Mera (Oleiros).

braiteiro.

Pontevedra: San Cosme de Barrelos. 


\section{jaugo.}

L e ón: Sabero.

sabú.

A stu ri as : Caldas de Oviedo, Caravia (Oviedo), Manzaneda (Oviedo), Pola de Laviana, Tudela de Veguín (Oviedo), Villaoril (Cangas de Narcea).

sabubina.

Asturias: Argame (Morcfn).

sabubo.

A st u ri as: Celorio (Llanes), Morcín, Villamejín (Proaza).

sabucal.

L e ó n : Castillo de la Valduerna (La Bañeza).

sabuco.

Ore n se: Villamartín de Valdeorras.

A s tu ri a s: Barzana de Quirós, Caravia, Corao (Cangas de Onís), Cornellana (Salas), Llanes, La Manjorga (Oviedo), Navia, Oviedo, Piedeloro (Carreño), Puente de los Fierros, Ribadesella, Sama de Langreo, Ujo (Mieres).

L e ó n: Cacabelos, Cimanes del Tejar, Magaz, Matalauenga

(Las Omañas), Narayola (Camponaraya), Paladín (Las Omañas), Secarejo (Cinanes del Tejar), Soto y Amio, Trascastro, La Utreta (Valdesamario), Villadepalos (Carracedelo), Villafranca del Bierzo, Villaviciosa de la Ribera (Llamas de la Ribera).

sabugal.

Asturias: Teverga. 
sabugo.

Cor uñ a: Boimorto, Teijeiro (Curtis).

L u g o: Baamonde (Begonte), Carlín (Friol), Castelo (Lugo), Condes (Friol), Corgo, Foilevar (Sarria), Lugo, Monterroso, Nadela (Lugo), Las Peares (Lugo), Puertomarín, Santa Eulalia de Sisoy (Cospeito), Santa Marina (Incio).

Pontevedra: Ventosa (Lalín).

A sturias: La Arena (Soto del Barco), Arbeyales (Somiedo), Arnao (Castrillón), Ayones (Luarca), Calleras (Tineo), Cangas de Narcea, Cangas de Onís, Cangas de Tineo, Cañedo (Grado), Celorio (Llanes), Colloto, Cordovero (Pravia), Cornellana (Salas), Forniellas (Allande), Grado, Latores (Oviedo), Lillo (Oviedo), Mallecina (Salas), Mieres, Obona (Tineo), Pereda (Oviedo), Prada (Allande), Prado (Teverga), Pravia, Priero (Salas), Quintana (Belmonte), La Reigada (Allande), Ribadesella, Ribera de Arriba, Sabiencia (Somiedo), Salinas (Castrillón), San Esteban de Pravia, San Facundo ('Tineo), Santa Eulalia (Tineo), San Tirso (Candamo), Santullano (Salas), Las Segadas (Oviedo), Valle del Ajo (Somiedo), Villamar, Villamejín (Proaza).

L e ó $\mathrm{n}$ : región de Babia, región de Cabrera, Castro de Lomba (Campo de la Lomba), Ponjos (Valdesamario), Trascastro (Peranzanes), Villablino, Villadecanes del Bierzo.

\section{sabugueiro.}

Cor uñ a: Arzúa, Beigondo (Santirso), Betanzos, Pastoriza a Vella (Arzúa), Pastoriza a Nova (Arzúa).

L u g o: Atán (Pantón), Brollón (Puebla del Brollón), Bustelo (Becerreá), Canelo (Castro del Rey), Lugo, Pena, San Pelayo de Arcos (Castroverde), Santalla (Lugo), Santa María (Sarria), Sarria, Vilacha (Becerreá).

Or e n s e: Allariz, Amoeiro, Baños de Molgas (Orense), Beiro (Carballeda), Bentraces (Barbadanes), Bouza (Orense), 
Junquera de Ambia, Maceda, La Merca, Loueiro (Amoeiro), Pereiro (Canedo), Petín, Ribadavia, La Rúa, Trasalva (Amoeiro).

L e ó n : región de Babia, región de Bierzo, región de la Cabrera, Ponferrada.

sabuguero.

L e ó n : Bembibre, región del Bierzo, Castro de Lomba (Campo de la Lomba), Fuentesnuevas (Ponferrada).

sabujo.

Cor ũ a : Boimorto, Curtis.

sabuquero.

L éón: Bierzo.

subuxeiro.

Pontevedra: Sotomayor (Vigo).

sagú.

Asturias: Villanueva (Ribadedeva).

sagugu.

Asturias: Celorio (Llanes), Tielve (Cabrales).

sambenito.

Asturias: Pola de Siero, Vigil (Siero).

samugueiro.

Pontevedra: Cotobad. 
saú.

A sturi a s: Tudela de Agüeria (Oviedo), Valdesoto (Siero), Vigil (Siero) ${ }^{\mathbf{1}}$.

saubú.

Asturias: Cayés (Llanera) ${ }^{2}$. saucero.

Cor u ̃̃ a Ferrol.

sauco.

A s turias: Avilés, Cangas de Onís, Lugo de Llanera (Llanera), Muñas de Abajo (Luarca), Oviedo, Santa Cruz de Mieres (Mieres), Santo Domingo (Avilés),

L e ó n : Quintana del Monte (Valdepolo), Valencia de don Juan, Villalquite (Valdepolo.)

saugo.

A s t u ri a s: Collanzo (Aller), Llamas (Aller), Pola de Siero, Trasmonte (Las Regueras), Viella (Siero).

L, éón : Oseja.

saugueiro.

Cor ũ̃ a: Guisamo (Bergondo), Sisán.

sáuquero.

L e ó n : Santibáñez de la Lomba (Campo de la Lomba).

siabugo.

Asturias: La Manjoya (Oviedo).

1 En los dos últimos lugares, sólo "flor de saili.

2 Se dice únicamente "flor de saubiu. 
siauco.

L e ó n : Oseja.

sungo.

L u g o: Mondoñedo.

suúcu.

Asturias: Pola de Siero.

xabú ${ }^{1}$.

Asturias: Ablaña (Mieres), Aller, Bello, Bóo (Aller), Cabañaquinta (Aller), Campomanes (Lena), Celleruelo (Laviana), Cenera (Nieres), El Condado (Oviedo), Entralgo (Laviana), El Entrego (Pola de Laviana), Herías (Lena), Iguanzo (Laviana), Lorio (Laviana), Mieres, Moreda (Aller) Muñera (Laviana), Murias (Aller), Parana (Lena), La Pereda (Mieres), Piñeres (Laviana), Pola de Laviana, Puente de Arco (Laviana), Ribota (Laviana), Santa Cruz (Llanera), Santullano (Mieres), Tudela de Agüeria (Oviedo), Turón (Mieres), Ujo (Mieres).

xabucu.

A st $u$ r i a : Pola de Laviana, Sueros (Mieres), 'Turón (Mieres), Ubriendes (Mieres).

xabugo.

Asturias: Collía (Parres), Doriga (Salas), Infiesto (Piloña), Mieres, Proaza, Quirós.

xabugueiro.

L $u$ g o: Eiras (Páramo).

1 Con $x$ represento en este indice a la consonante prepalatal fricativa sorda. 
RPE, XI,I, I957 DERTVADOS DE "SABUCUS" EN LA TOPONIMIA...

xagú.

A sturia s: Ablaña (Mieres), Huería de San Tirso (Mieres), Huería de San Juan (Mieres), Sueros (Mieres).

\section{xagugo.}

Asturias: Grado.

\section{xagüina.}

Asturias: Zureda (Lena).

\section{xaugu.}

Asturias: Amieva, Parres.

xiabiú.

Asturias: Laviana.

\section{xuủcu.}

Asturias: Rozadas (Villaviciosa).

Manuel, Alvar.

Universidad de Granada. 